\title{
Forecasting the solar activity cycle: new insights
}

\author{
Dibyendu Nandy ${ }^{1}$ and Bidya Binay Karak ${ }^{2}$ \\ ${ }^{1}$ Indian Institute for Science Education and Research, Kolkata, Mohanpur 741252, \\ West Bengal, India \\ ${ }^{2}$ Department of Physics, Indian Institute of Science, Bangalore 560012, India
}

\begin{abstract}
Having advance knowledge of solar activity is important because the Sun's magnetic output governs space weather and impacts technologies reliant on space. However, the irregular nature of the solar cycle makes solar activity predictions a challenging task. This is best achieved through appropriately constrained solar dynamo simulations and as such the first step towards predictions is to understand the underlying physics of the solar dynamo mechanism. In BabcockLeighton type dynamo models, the poloidal field is generated near the solar surface whereas the toroidal field is generated in the solar interior. Therefore a finite time is necessary for the coupling of the spatially segregated source layers of the dynamo. This time delay introduces a memory in the dynamo mechanism which allows forecasting of future solar activity. Here we discuss how this forecasting ability of the solar cycle is affected by downward turbulent pumping of magnetic flux. With significant turbulent pumping the memory of the dynamo is severely degraded and thus long term prediction of the solar cycle is not possible; only a short term prediction of the next cycle peak may be possible based on observational data assimilation at the previous cycle minimum.
\end{abstract}

Keywords. Sun: activity, Sun: magnetic fields, sunspots.

\section{Introduction}

The solar cycle is not regular. The individual cycles vary in strength from one cycle to another. Therefore prediction of future cycles is a non-trivial task. However forecasting future cycle amplitudes is important because of the impact of solar activity on our space environment. Unfortunately, recent efforts to predict the solar cycle did not reach any consensus, with a wide range of forecasts for the strength of the ongoing cycle 24 (Pesnell 2008).

Kinematic dynamo models based on the Babcock-Leighton mechanism has proven to be a viable approach for modeling the solar cycle (e.g., Muñoz-Jaramillo et al. 2010; Nandy 2011; Choudhuri 2013). In such models, the poloidal field is generated from the decay of tilted active regions near the solar surface mediated via near-surface flux transport processes. In this model the large-scale coherent meridional circulation plays a crucial role (Choudhuri et al. 1995; Yeates, Nandy \& Mackay 2008; Karak 2010; Nandy, MuñozJaramillo \& Martens 2011; Karak \& Choudhuri 2012). This is because the meridional circulation is believed to transport the poloidal field - generated near the solar surface - to the interior of the convection zone where the toroidal field is generated through stretching by differential rotation. The time necessary for this transport introduces a memory in the solar dynamo, i.e., the toroidal field (which gives rise the sunspot eruptions) has an in-built "memory" of the earlier poloidal field. Yeates, Nandy \& Mackay (2008) systematically studied this issue and showed that in the advection-dominated regime of the dynamo the poloidal field is mainly transported by the meridional circulation and the solar cycle memory persists over many cycles (see also Jiang, Chatterjee \& 
Choudhuri 2007). On the other hand, in the diffusion-dominated regime of the dynamo, the poloidal field is mainly transported by turbulent diffusion and the memory of the solar cycle is short - roughly over a cycle. Recent studies favor the diffusion-dominated solar convection zone (Miesch et al. 2011) and the diffusion-dominated dynamo is successful in modeling many important aspects of the solar cycle including the Waldmeier effect and the grand minima (Karak \& Choudhuri 2011; Choudhuri \& Karak 2009; Karak 2010; Choudhuri \& Karak 2012; Karak \& Petrovay 2013).

Using an advection dominated B-L dynamo Dikpati de Toma \& Gilman (2006) predicted a strong cycle 24. On the other hand, Choudhuri et al. (2007) used a diffusiondominated model and predicted a weak cycle (see also Jiang et al. 2008). However in most of the models, particularly in these prediction models, the turbulent pumping of magnetic flux - an important mechanism for transporting magnetic field in the convection zone - was ignored. Theoretical as well as numerical studies have shown that a horizontal magnetic field in the strongly stratified turbulent convection zone is pumped preferentially downward towards the base of the convection zone (stable layer) and a few $\mathrm{m} / \mathrm{s}$ pumping speed is unavoidable in many convective simulations (e.g., Petrovay \& Szakaly 1993; Brandenburg et al. 1996; Tobias et al. 2001; Dorch \& Nordlund 2001; Ossendrijver et al. 2002; Käpylä et al. 2006; Racine et al. 2011). Recently, we have studied the impact of turbulent pumping on the memory of the solar cycle and hence its relevance for solar cycle forecasting (Karak \& Nandy 2012). Here we provide a synopsis of our findings and discuss its implications for solar cycle predictability.

\section{Model}

The evolution of the magnetic fields for a kinematic $\alpha \Omega$ dynamo model is governed by the following two equations.

$$
\begin{aligned}
& \frac{\partial A}{\partial t}+\frac{1}{s}(\mathbf{v} \cdot \nabla)(s A)=\eta_{p}\left(\nabla^{2}-\frac{1}{s^{2}}\right) A+\alpha B \\
& \frac{\partial B}{\partial t}+\frac{1}{r}\left[\frac{\partial}{\partial r}\left(r v_{r} B\right)+\frac{\partial}{\partial \theta}\left(v_{\theta} B\right)\right]=\eta_{t}\left(\nabla^{2}-\frac{1}{s^{2}}\right) B+s\left(\mathbf{B}_{p} \cdot \nabla\right) \Omega+\frac{1}{r} \frac{d \eta_{t}}{d r} \frac{\partial}{\partial r}(r B)
\end{aligned}
$$

with $s=r \sin \theta$. Here $A$ is the vector potential of the poloidal magnetic field, $B$ is the toroidal magnetic field, $\mathbf{v}=v_{r} \hat{r}+v_{\theta} \hat{\theta}$ is the meridional circulation, $\Omega$ is the internal angular velocity, $\alpha$ is the source term for the poloidal field by the B-L mechanism and $\eta_{p}, \eta_{t}$ are the turbulent diffusivities for the poloidal and toroidal components. With the given ingredients, we solve the above two equations to study the evolution of the magnetic field in the dynamo model. The details of this model can be found in Nandy \& Choudhuri (2002) and Chatterjee, Nandy \& Choudhuri (2004). However for the sake of comparison with earlier results we use similar parameters as in Yeates, Nandy \& Mackay (2008).

In the mean-field induction equation, the turbulent pumping naturally appears as an advective term. Therefore to include its effect in the present dynamo model, we include the turbulent pumping term shown by the following expression in the advection term of the poloidal field equation (Eq. 2.1).

$$
\gamma_{r}=-\gamma_{0 r}\left[1+\operatorname{erf}\left(\frac{\mathrm{r}-0.715}{0.015}\right)\right]\left[1-\operatorname{erf}\left(\frac{\mathrm{r}-0.97}{0.1}\right)\right]\left[\exp \left(\frac{\mathrm{r}-0.715}{0.25}\right)^{2} \cos \theta+1\right]
$$

where $\gamma_{0 r}$ determines the strength of the pumping which we vary in our simulations. Note 
that we introduce pumping only in the poloidal field because turbulent pumping is likely to be relatively less effective on the toroidal component (e.g., Käpylä et al. 2006). The toroidal field is stronger, intermittent and subject to buoyancy forces and therefore it is less prone to be pumped downwards. Also note that we do not consider any latitudinal pumping.

To study the solar cycle memory we have to allow for irregularities in the cycle amplitude through the introducing of stochasticity in the model. Presently we believe that there are two important sources of randomness in the solar dynamo - stochastic fluctuations in the B-L process of generating the poloidal field and stochastic fluctuations in the meridional circulation. In this work, we introduce stochastic fluctuations in the B-L $\alpha$-effect appearing in Eq. 2.1 to capture the irregularity in the B-L process of poloidal field generation. We set $\alpha_{0}=\alpha_{\text {base }}+\alpha_{\text {fluc }} \sigma\left(t, \tau_{\text {cor }}\right)$. Throughout all the calculations we take $\alpha_{\text {fluc }}=\alpha_{\text {base }}=30 \mathrm{~m} \mathrm{~s}^{-1}$ (i.e., $100 \%$ level of fluctuations). The coherence time $\tau_{c o r}$ is chosen to allow for about 10 fluctuations in each cycle. In doing so, the value of $\tau_{\text {cor }}$ typically ranges between $0.5 \mathrm{yr}$ to $2.0 \mathrm{yr}$ which is consistent with the surface flux transport process in the B-L mechanism.

\section{Results}

We have carried out extensive simulations with stochastically varying $\alpha$ at different downward pumping speeds ranging from $0-4 \mathrm{~m} \mathrm{~s}^{-1}$. We have performed simulations in two different regimes of the dynamo -the diffusion-dominated regime with parameters $v_{0}=15 \mathrm{~m} \mathrm{~s}^{-1}, \eta_{0}=1 \times 10^{12} \mathrm{~cm}^{2} \mathrm{~s}^{-1}$ and the advection-dominated regime with $v_{0}=$ $26 \mathrm{~m} \mathrm{~s}^{-1}, \eta_{0}=1 \times 10^{12} \mathrm{~cm}^{2} \mathrm{~s}^{-1}$. In the previous case diffusive transport of the poloidal magnetic field is more important compared to advective transport by meridional flow whereas in the latter case it is the other way around.

While there are some effect of turbulent pumping on the solar cycle period and the latitudinal distribution of the magnetic field (which have already been explored by Guerrero \& de Gouveia Dal Pino 2008) we are primarily interested in the relationship between the poloidal field at solar minima and the toroidal field of subsequent cycles. To study this we compute the correlation between the peak of the surface radial flux $\left(\phi_{r}\right)$ of cycle $n$ with that of the deep-seated toroidal flux $\left(\phi_{t o r}\right)$ of different cycles. Here we consider $\phi_{r}$ as the flux of radial field over the solar surface from latitude $70^{0}$ to $89^{0}$, and $\phi_{t o r}$ as the flux of toroidal field over the region $r=0.677-0.726 R$ and latitude $10^{0}$ to $45^{0}$. In table 1, we present the Spearman's rank correlation coefficients and significance levels in two different regimes with increasing pumping speed. From this table it is evident that in the advection-dominated regime, in absence of pumping, the polar flux of cycle $n$ correlates with the toroidal flux of cycle $n+1, n+2$ and $n+3$, whereas in the diffusion dominated regime only one cycle correlation exists (i.e., the polar flux of cycle $n$ correlates only with the toroidal flux of cycle $n+1)$. The underlying reason for this difference was already discussed in Yeates, Nandy \& Mackay (2008). However, it is significant that with the increase in the pumping speed in the advection-dominated region, the higher order correlations slowly diminish and with a $2.0 \mathrm{~m} \mathrm{~s}^{-1}$ pumping speed only the $n$ to $n+1$ correlation exists and other correlations are destroyed. However the behavior in the diffusion-dominated regime remains qualitatively unchanged.

Fig. 1 shows the correlation plot with $2.0 \mathrm{~m} \mathrm{~s}^{-1}$ pumping amplitude for the advectiondominated regime whereas Fig. 2 shows the same for the diffusion-dominated case. We also find that with increasing strength of turbulent pumping the $n$ to $n+1$ correlations 
Table 1. Correlation coefficients $\left(r_{s}\right)$ and percentage significance levels $(p)$ for peak surface radial flux $\Phi_{\text {r }}$ of cycle $n$ versus peak toroidal flux $\Phi_{\text {tor }}$ of different cycles for 275 solar cycles data. The first column denotes the amplitude of the turbulent pumping speed in various simulation studies. The top row corresponds to the case without turbulent pumping and subsequent rows correspond to simulations with increasing pumping speeds.

\begin{tabular}{llrr}
\hline & & Dif. Dom. & Adv. Dom. \\
\hline Pumping & \multicolumn{1}{c}{ Parameters } & $r_{s}(p)$ & $r_{s}(p)$ \\
\hline & $\Phi_{\mathrm{r}}(n) \& \Phi_{\text {tor }}(n)$ & $0.19(99.9)$ & $0.57(99.9)$ \\
$0 \mathrm{~m} \mathrm{~s}^{-1}$ & $\Phi_{\mathrm{r}}(n) \& \Phi_{\text {tor }}(n+1)$ & $0.64(99.9)$ & $0.77(99.9)$ \\
& $\Phi_{\mathrm{r}}(n) \& \Phi_{\text {tor }}(n+2)$ & $0.04(55.9)$ & $0.46(99.9)$ \\
& $\Phi_{\mathrm{r}}(n) \& \Phi_{\text {tor }}(n+3)$ & $0.22(99.9)$ & $0.27(99.9)$ \\
\hline & $\Phi_{\mathrm{r}}(n) \& \Phi_{\text {tor }}(n)$ & $-0.06(67.0)$ & $0.41(99.9)$ \\
& $\Phi_{\mathrm{r}}(n) \& \Phi_{\text {tor }}(n+1)$ & $0.67(99.9)$ & $0.72(99.9)$ \\
& $\Phi_{\mathrm{r}}(n) \& \Phi_{\text {tor }}(n+2)$ & $0.09(83.9)$ & $0.29(99.9)$ \\
& $\Phi_{\mathrm{r}}(n) \& \Phi_{\text {tor }}(n+3)$ & $-0.02(26.5)$ & $-0.01(18.9)$ \\
\hline \multirow{4}{*}{$2 \mathrm{~m} \mathrm{~s}^{-1}$} & $\Phi_{\mathrm{r}}(n) \& \Phi_{\text {tor }}(n)$ & $0.12(94.9)$ & $0.19(99.8)$ \\
& $\Phi_{\mathrm{r}}(n) \& \Phi_{\text {tor }}(n+1)$ & $0.43(99.9)$ & $0.75(99.9)$ \\
& $\Phi_{\mathrm{r}}(n) \& \Phi_{\text {tor }}(n+2)$ & $-0.16(99.9)$ & $0.07(73.8)$ \\
& $\Phi_{\mathrm{r}}(n) \& \Phi_{\text {tor }}(n+3)$ & $-0.02(20.8)$ & $-0.10(89.8)$ \\
\hline & $\Phi_{\mathrm{r}}(n) \& \Phi_{\text {tor }}(n)$ & $0.11(49.2)$ & $0.29(92.0)$ \\
& $\Phi_{\mathrm{r}}(n) \& \Phi_{\text {tor }}(n+1)$ & $0.32(99.9)$ & $0.62(99.9)$ \\
& $\Phi_{\mathrm{r}}(n) \& \Phi_{\text {tor }}(n+2)$ & $-0.18(99.6)$ & $0.07(78.0)$ \\
& $\Phi_{\mathrm{r}}(n) \& \Phi_{\text {tor }}(n+3)$ & $0.03(36.6)$ & $-0.10(91.6)$ \\
\hline \multirow{3}{*}{$4 \mathrm{~m} \mathrm{~s}^{-1}$} & $\Phi_{\mathrm{r}}(n) \& \Phi_{\text {tor }}(n)$ & $0.19(99.8)$ & $0.30(99.9)$ \\
& $\Phi_{\mathrm{r}}(n) \& \Phi_{\text {tor }}(n+1)$ & $0.26(99.9)$ & $0.46(99.9)$ \\
& $\Phi_{\mathrm{r}}(n) \& \Phi_{\text {tor }}(n+2)$ & $-0.16(99.3)$ & $0.07(72.8)$ \\
& $\Phi_{\mathrm{r}}(n) \& \Phi_{\text {tor }}(n+3)$ & $-0.10(91.9)$ & $-0.22(99.9)$ \\
\hline
\end{tabular}

also decrease rapidly in both the advection and in the diffusion-dominated regime (see Table 1).

\section{Conclusion and Discussion}

We have introduced turbulent pumping of the magnetic flux in a B-L type kinematic dynamo model and have carried out several extensive simulations with stochastic fluctuation in the B-L $\alpha$ with different strengths of downward turbulent pumping in both advection- and diffusion-dominated regimes of the solar dynamo. We find that multiple cycle correlations between the surface polar flux and the deep-seated toroidal flux in the advection-dominated dynamo model degreades severely when we introduce turbulent pumping. With $2 \mathrm{~m} \mathrm{~s}^{-1}$ as the typical pumping speed, the timescale for the poloidal field to reach the base of the convection zone is about 4 years, which is even shorter than the timescale of turbulent diffusion (and much shorter than the advective timescale due to meridional circulation). Consequently the behavior found in the advection-dominated dynamo model with pumping is similar to that seen in the diffusion-dominated dynamo model indicating that downward turbulent pumping short-circuits the meridional flow transport loop for the poloidal flux. This transport loop is first towards the poles at near-surface layers and then downwards towards the deeper convection zone and subsequently equatorwards. However, when pumping is dominant, then the transport loop is predominantly downwards straight into the interior of the convection zone. 


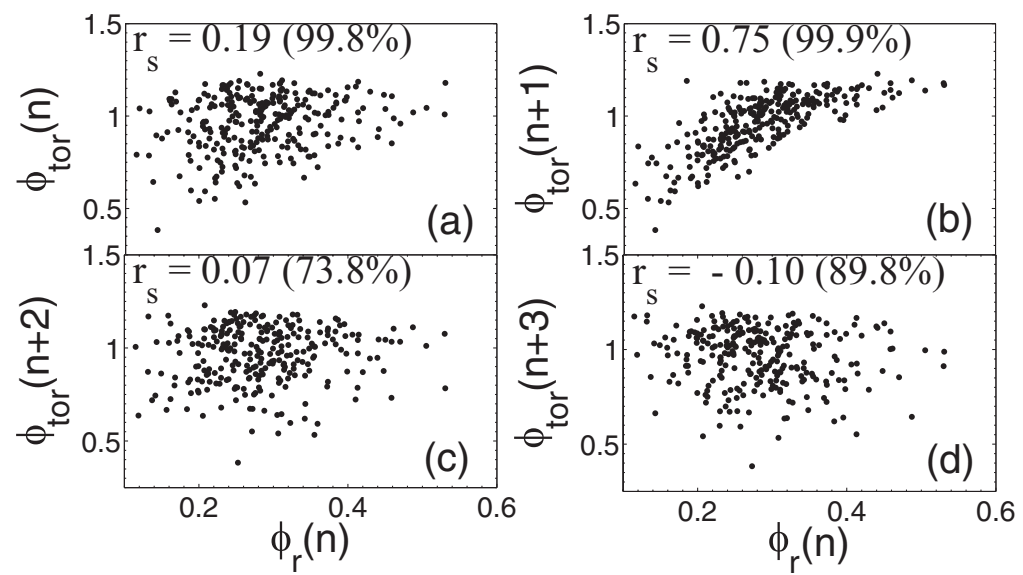

Figure 1. Scattered plots of the peak (polar) radial flux $\phi_{\mathrm{r}}(n)$ and the peak (deep-seated) toroidal flux $\phi_{\text {tor }}$ of cycle (a) $n$ (b) $n+1$, (c) $n+2$, and (d) $n+3$ in the advection-dominated regime with a pumping speed amplitude of $2 \mathrm{~m} \mathrm{~s}^{-1}$. The flux values are in units of $10^{25} \mathrm{Mx}$. The Spearman's rank correlation coefficients $\left(r_{s}\right)$ along with significance levels are inscribed. Reproduced from Karak \& Nandy (2012).
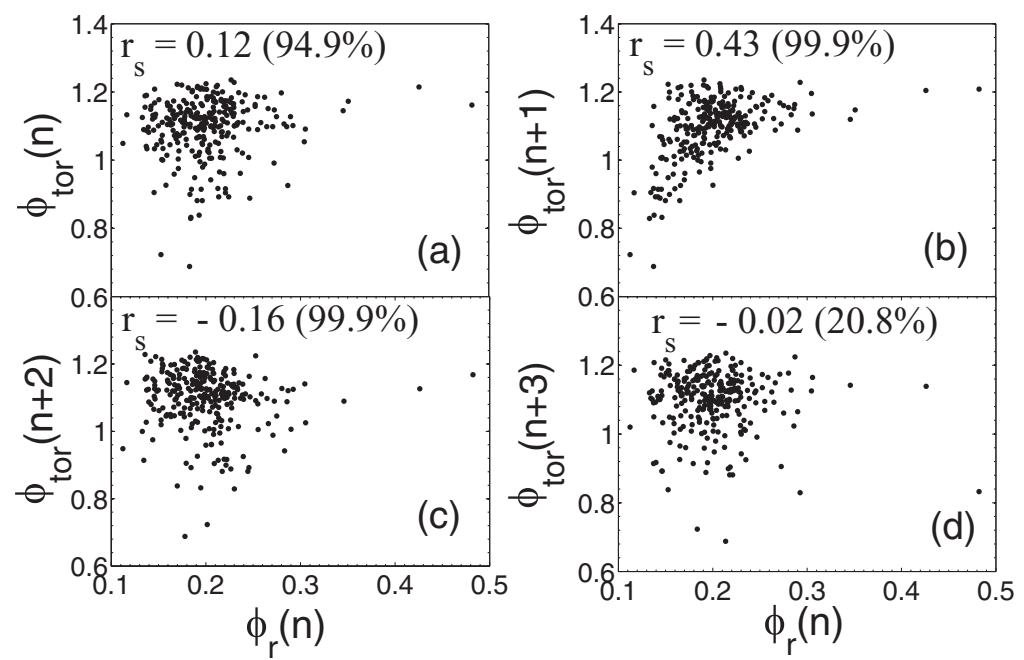

Figure 2. Same as Fig. 2, but for the diffusion-dominated regime. Reproduced from Karak \& Nandy (2012).

An interesting and somewhat counter-intuitive possibility that our findings raise is that the solar convection zone may not be diffusion-dominated, or advection-dominated, but rather be dominated by turbulent pumping. Note that this does not rule out the possibility that in the stable layer beneath the base of the convection zone, meridional circulation still plays an important and dominant role in the equatorward transport of toroidal flux and thus, in generating the butterfly diagram.

Our result implies with turbulent pumping as the dominant mechanism for flux transport, the solar cycle memory is short. This short memory, lasting less than a complete 11 year cycle implies that solar cycle predictions for the maxima of cycles are best achieved at the preceding solar minimum, about 4-5 years in advance and long-term predictions are unlikely to be accurate. This also explains why early predictions for the amplitude 
of solar cycle 24 were inaccurate and generated a wide range of results with no consensus. The lesson that we take from this study is that it is worthwhile to invest time and research to understand the basic physics of the solar cycle first, and that advances made in this understanding will lead to better forecasting capabilities for solar activity.

\section{Acknowledgements}

The authors would like to thank the Ministry of Human Resource Development and the Department of Science and Technology, Government of India, for funding this research summarized here.

\section{References}

Brandenburg, A. Jennings, R. L., Nordlund, Å., Rieutord, M., Stein, R. F., \& Tuominen, I. 1996, Journal Fluid Mech., 306, 325

Chatterjee, P., Nandy, D., \& Choudhuri, A. R. 2004, A\&\&A, 427, 1019

Choudhuri, A. R. 2013, these proceedings (arXiv:1211.0520)

Choudhuri, A. R., Chatterjee, P., \& Jiang, J. 2007, Phys. Rev. Lett., 98, 1103

Choudhuri, A. R. \& Karak, B. B. 2009, RAA, 9, 953

Choudhuri, A. R. \& Karak, B. B. 2012, Phys. Rev. Lett., 109, 171103

Choudhuri, A. R., Schüssler, M., \& Dikpati, M. 1995, A\&AA, 303, L29

Dorch, S. B. F. \& Nordlund, A.. 2001, A\&̈A 365, 562.

Dikpati, M. de Toma, G., \& Gilman, P. A. 2006, Geophys. Res. Lett., 33, L05102

Guerrero, G. \& de Gouveia Dal Pino, E. M. 2008, ApJ 485, 267

Jiang, J., Chatterjee, P., \& Choudhuri, A. R. 2007, MNRAS, 381, 1527

Käpylä, P. J., Korpi, M. J., Ossendrijver, M., \& Stix, M. 2006, A\&SA 455, 401

Karak, B. B. 2010, ApJ, 724, 1021

Karak, B. B. \& Choudhuri, A. R. 2011, MNRAS, 410, 1503

Karak, B. B. \& Choudhuri, A. R. 2012, Solar Phys., 278, 137

Karak, B. B. \& Nandy, D. 2012, ApJ, 761, L13

Karak, B. B. \& Petrovay, K. 2013, Solar Phys., 282, 321

Miesch, M. S., Featherstone, N. A., Rempel, M., \& Trampedach, R. 2012, ApJ, 757, 128

Muñoz-Jaramillo, A, Nandy, D., Martens, P. C. H.., \& Yeates, A. R. 2010, ApJ 720, L20

Nandy, D. 2012, IAU Symp. 286, Comparative Magnetic Minima: Characterizing Quiet Times in the Sun and Stars, ed. C. H. Mandrini \& D. F. Webb (Cambridge: Cambridge Univ. Press), 54

Nandy, D. \& Choudhuri, A. R. 2002, Science, 296, 1671

Nandy, D., Muñoz-Jaramillo, A., \& Martens, P. C. H. 2011, Nature, 471, 80

Ossendrijver, M., Stix, M., Brandenburg, A., \& Rüdiger, G. 2002, ApJ 394, 735

Petrovay, K. \& Szakaly, G. 1993, A\&A 274, 543

Pesnell, W. D. 2008, Solar Phys., 252, 209.

Racine, È., Charbonneau, P., Ghizaru, M., Bouchat, A., \& Smolarkiewicz, P. K. 2011, ApJ 735, 46

Tobias, S. M. et al. 2001, ApJ 549, 1183

Yeates, A. R., Nandy, D., \& Mackay, D. H. 2008, ApJ, 673, 544 